\title{
Alterstice
}

Revue internationale de la recherche interculturelle

International Journal of Intercultural Research

Revista International de la Investigacion Intercultural

\section{Violence conjugale, diversité ethnique et changements politiques et économiques dans une communauté des hautes terres au Vietnam [synthèse en français]}

\section{Lynn Kwiatkowski}

Volume 3, numéro 2, 2013

Violence conjugale et diversité culturelle

URI : https://id.erudit.org/iderudit/1077521ar

DOI : https://doi.org/10.7202/1077521ar

Aller au sommaire du numéro

Éditeur(s)

Alterstice

ISSN

1923-919X (numérique)

Découvrir la revue

Citer ce document

Kwiatkowski, L. (2013). Violence conjugale, diversité ethnique et changements politiques et économiques dans une communauté des hautes terres au Vietnam [synthèse en français]. Alterstice, 3(2), vi-viii. https://doi.org/10.7202/1077521ar 


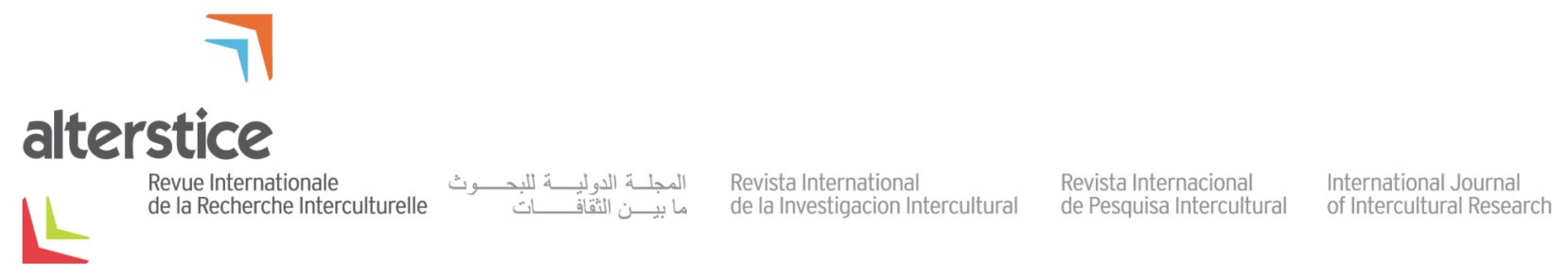

ARTICLE THÉMATIQUE

\title{
Violence conjugale, diversité ethnique et changements politiques et économiques dans une communauté des hautes terres au Vietnam [synthèse en français*]
}

Lynn Kwiatkowski ${ }^{1}$

\author{
Rattachement de l'auteure \\ ${ }^{1}$ Department of Anthropology, Colorado State University, États-Unis
}

\section{Correspondance}

lynn.kwiatkowski@colostate.edu

\section{Mots clés}

violence conjugale; ethnicité; économie politique de la violence conjugale; Vietnam

\section{Pour citer cet article :}

Kwiatkowski, L. (2013). Violence conjugale, diversité ethnique et changements politiques et économiques dans une communauté des hautes terres au Vietnam [synthèse en français*]. Alterstice, 3(2), i-iii.

*L'article complet en anglais est disponible sur le site d'Alterstice.

\section{Introduction}

$\mathrm{Au}$ Vietnam, la violence conjugale touche une variété de groupes ethniques dans différents contextes sociaux, culturels et environnementaux. Cet article examine le rôle perçu de la culture sur les manifestations de la violence conjugale au sein de différents groupes ethniques vivant dans une même communauté au Vietnam, en lien avec les influences sociales structurelles sur cette forme de violence sexiste. L'article traite des perceptions de la violence à l'égard des femmes des membres de trois groupes ethniques (les Kinh, les Muong et les Dao) qui vivent dans une communauté isolée dans une province au nord du pays.

\section{Objectifs}

Dans cet article, nous définissons premièrement la place occupée par la violence conjugale au sein des groupes ethniques évoluant dans un environnement social, politique et économique en changement, en examinant les raisons qui justifient la violence des hommes à l'égard de leurs femmes aux yeux des membres de ces groupes. Ensuite, nous évaluons les problèmes auxquels font toujours face les femmes victimes de mauvais traitements, malgré l'adoption d'une nouvelle loi sur la violence conjugale et les nouvelles orientations des professionnels et des dirigeants communautaires en matière de prestation de services pour les femmes maltraitées. 


\section{Méthodologie}

Cet article se base sur mes recherches ethnographiques qualitatives sur le terrain axées sur la violence conjugale, en particulier la violence envers les femmes, sur une période de deux mois et demi. Cette recherche comportait une observation participante dans la communauté des hautes terres et des entretiens formels, approfondis, semistructurés et non directifs avec des représentants de 39 familles des trois groupes ethniques, 15 femmes de chacun des groupes ethniques ayant été victimes de violence conjugale avant ou pendant la recherche et 24 représentants de l'État ou acteurs non étatiques, dirigeants soutenus par l'État, professionnels de la santé, guérisseurs locaux et autres informateurs clés vivant dans les deux communes étudiées. Nous avons également recueilli du matériel, des rapports, des données et des documents pertinents provenant d'organismes gouvernementaux, d'organisations communautaires soutenues par l'État, d'organisations non gouvernementales locales et internationales et des médias.

Cette étude qualitative s'appuie sur le cadre théorique de l'économie politique de la violence conjugale. Ce cadre vise à étudier la manière dont les forces culturelles, sociales, politiques et économiques dans la communauté des hautes terres - et plus généralement au Vietnam - ont influencé les expériences et les perceptions de la violence conjugale des membres des trois groupes ethniques, et les réactions des dirigeants communautaires quant à cette forme de violence sexiste. Selon Adelman (2004), cette approche « situe la violence conjugale dans un contexte historique et culturel pour révéler l'intersection entre la violence conjugale et 1) l'organisation du régime politique; 2) l'aménagement de l'économie; 3) l'idéologie familiale dominante exprimée de manière normative dans les politiques de l’État. » (p. 46)

\section{Résultats}

Malgré le rôle important de la culture, quelques-uns seulement des dirigeants locaux et des guérisseurs avec qui nous nous sommes entretenus dans cette communauté percevaient ou ont rapporté des différences importantes dans les expériences de violence conjugale des membres de chaque groupe ethnique. À l'inverse, les forces politiques et économiques semblent avoir une influence plus grande sur les expériences de violence conjugale des femmes membres de ces groupes que les différences culturelles entre groupes ethniques. Ces forces politiques et économiques comprennent la transition d'une économie planifiée à une économie d'orientation socialiste amorcée dans les années 1980, l'adoption d'une nouvelle loi sur la violence conjugale dans le contexte de changement économique et social et un changement d'orientation de l'État envers les groupes ethniques minoritaires. De plus, l'intersection entre les approches du gouvernement vietnamien vis-à-vis des groupes ethniques minoritaires, le manque de barrières distinctes entre les groupes ethniques et l'interaction sociale à long terme des membres de ces trois groupes ethniques dans la communauté des hautes terres pourraient avoir amené les dirigeants locaux et les guérisseurs à percevoir le problème contemporain de violence conjugale dans les groupes ethniques de leur communauté comme découlant principalement des forces structurelles sociales et des changements politiques et économiques à l'échelle générale plutôt que des caractéristiques culturelles propres à chaque groupe.

\section{Analyse}

À la lumière de cette découverte, cette étude soutient que, si les forces culturelles façonnent les pratiques et les expériences de violence conjugale, les forces structurelles sociales ont un rôle important et, dans certains cas, une influence encore plus grande que les forces culturelles sur les manifestations et les expériences de violence conjugale. Cette affirmation est illustrée par la difficulté que les femmes continuent à éprouver pour trouver des protections contre la violence de leur mari malgré l'adoption de la nouvelle loi sur la violence conjugale. Cette situation est attribuable en particulier aux pratiques du personnel de l'État qui minimisent la violence des hommes envers leurs femmes et à la pression exercée sur les femmes dans le cadre du processus de réconciliation mis en place par l'État afin que les femmes restent avec leur mari. Ces problèmes influent sur la vulnérabilité des femmes en situation de violence et ont des effets négatifs sur la santé et la vie sociale plus importants que les différences vécues en raison de l'identification à un groupe ethnique déterminé. 
Une communauté a mis sur pied un club novateur pour prévenir la violence conjugale. Les membres du club s'efforcent d'influencer les perceptions qu'ont les hommes et les femmes des différents groupes ethniques par rapport à la violence conjugale et de prévenir les nouveaux cas de violence domestique. Néanmoins, malgré ces nouvelles approches de prévention importantes, dont certaines ont été inspirées par des organisations internationales, malgré le processus de réconciliation du gouvernement toujours en place dans le cadre de la nouvelle loi sur la violence conjugale et malgré ces clubs, les femmes victimes de mauvais traitements demeurent vulnérables à la violence de leur mari.

\section{Conclusion}

Dans la communauté visée, les forces sociales structurelles s'entremêlent avec les conceptions culturelles communes et entretiennent ainsi la violence faite aux femmes par leur mari. Ces conceptions comprennent la supériorité des hommes sur leurs femmes inspirées par le confucianisme, le blâme jeté sur les femmes pour la violence de leur mari, l'appropriation et le soutien public par les femmes de cette idée et l'acceptation par les hommes et les femmes de l'idée que les maris possèdent un tempérament fort ou bouillant difficile à contrôler et exprimé par la violence. Cette étude avance que l'intersection entre le pouvoir du gouvernement, les processus structuraux et les conceptions culturelles communes sur les sexes, le mariage, la famille et la violence ont joué un rôle crucial pour façonner l'expérience des femmes victimes et des hommes acteurs de violence conjugale parmi les membres des trois groupes ethniques vivant dans la communauté visée par notre recherche.

La nouvelle loi sur la violence conjugale ne contribue pas clairement à évincer les approches traditionnelles de violence conjugale. Cependant, les fonctionnaires, et potentiellement quelques membres d'ONG locales ainsi que d'autres professionnels, auront l'occasion de s'exprimer sur ce qu'ils perçoivent comme des problèmes liés à la nouvelle loi sur la violence conjugale lors d'une évaluation quinquennale officielle et pourraient ainsi offrir une meilleure protection aux femmes victimes de violence.

\section{Références*}

Adelman, M. (2004). The battering state: Towards a political economy of domestic violence. Journal of Poverty 8(3), 45-64.

*Voir l'article en anglais pour la bibliographie d'ensemble. 